\title{
Annular lichen planus: clinical and dermoscopic features
}

\author{
Aicha Nassiri', Niema Aqil', Sara Elloudi', Hanane Baybay ${ }^{1}$, Fatima Zahra Mernissi', \\ Omar Amrani ${ }^{2}$, Mustapha Ahsaini ${ }^{2}$, Alaoui Nabil' ${ }^{2}$, Mohamed Fadl Tazi ${ }^{2}$, \\ Moulay El Hassan Farih ${ }^{2}$
}

\begin{abstract}
${ }^{1}$ Department of Dermatology, Hassan II University Hospital Center, Fes, Morocco, ${ }^{2}$ Department of Urology, Hassan II University Hospital Center, Fes, Morocco
\end{abstract}

Corresponding author: Dr. Aicha Nassiri, E-mail: aichanassiri6@gmail.com

Sir,

Annular lichen planus (ALP) is a long-recognized clinical variant of lichen planus, but is often considered uncommon in occurrence. The typical distribution and presentation of this variant have not been well described.

We sought to better define the sites affected and clinical characteristics of the annular variant of lichen planus, along with the age and race of patients affected with this disorder.

A 34-year-old man visited our department for evaluation of a three-month, mildly pruritic eruption on the genital area. Clinical examination violaceous plaques of annular morphology with central atrophyon scrotum (Fig. 1). Application of dermoscopy revealed white annular lines (the so-called Wickham striae) were dermoscopically evident, along with dotted and short linear vessels and yellow scales (Fig. 2). Since the dermoscopic presence of Wickham striae is considered highly specific of LP, the dermoscopic findings prompted us to perform a biopsy for histopathologic assessment. Histopathology, revealed hyperkeratosis, dense hypergranulosis, vacuolar degeneration of basal cell keratinocytes, band-like lymphocytic infiltration in the upper dermis, as well as presence of colloid bodies, justifying the diagnosis of LP.

Dermoscopy is a non-invasive tool that is widely recognized and used in the diagnosis of pigmented and non-pigmented skin tumors $[1,2]$. In recent years,

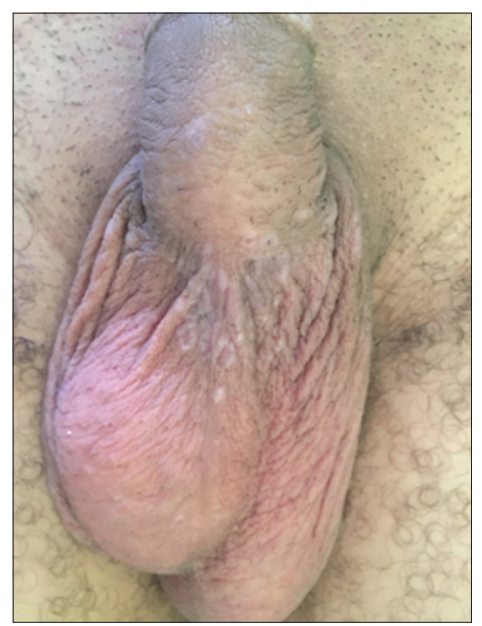

Figure 1: Violaceous plaques of annular morphology with central atrophyon scrotum.

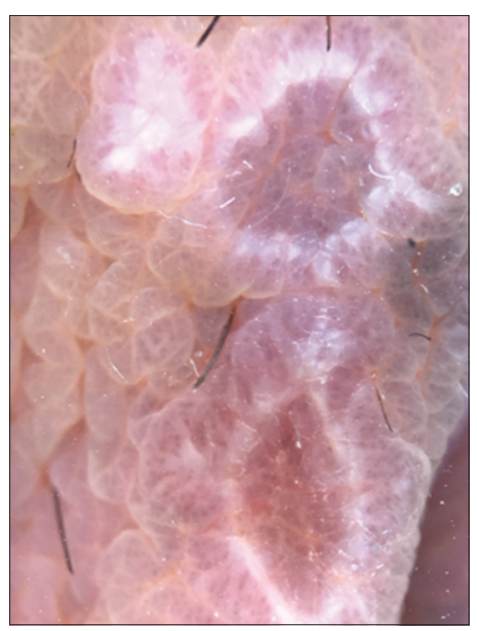

Figure 2: Annular Wickham striae.

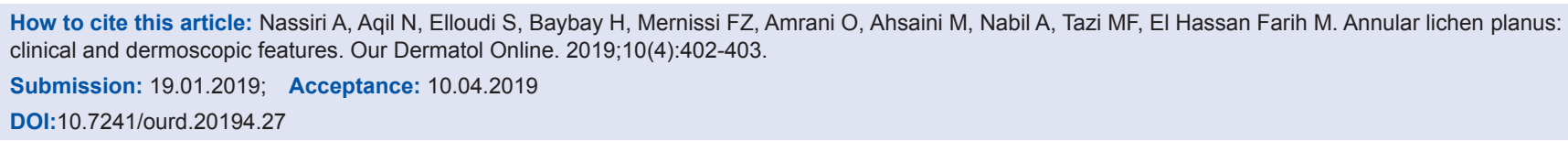


dermoscopy has been used for other dermatologic diseases including psoriasis, lichen planus, alopecia, and skin infestations $[1,2]$. Lichen planus (LP) is an acute or chronic inflammatory skin disorder characterized by discrete, violaceous, polygonal papules $[2,3]$. Though the diagnosis of LP can be made clinically, it can sometimes be challenging and histopathological examination is needed. Dermoscopic examination may be helpful in these settings to aid the diagnosis. In this study, we aimed to categorize the dermoscopic images of LP patients before and after treatment.

Wickham striae is commonly seen on dermoscopic examination in CLP lesions and it corresponds to hypergranulosis histologically [3-6]. WS disappears after treatment, suggesting that we can use it as an activation marker in LP lesions.

Wickham striae is classically seen as white crossing lines on dermoscopic evaluation and defined as "reticular pattern WS" [3,5].

In the current case, circular and radial streaming Wickham striae patterns were detected similar to the Tan et al. study. We believe that dermoscopic evaluation can be useful both in the diagnosis and follow up of LP.

ALP commonly involves the male genitalia but also has a predilection for intertriginous areas such as the axilla and groin folds. Eruptions typically consist of a few lesions localized to one or a few sites. Distal aspects of the extremities, and less commonly the trunk, may also be involved. ALP is a subtype of lichen planus that may be more common than is reflected in the literature.

\section{Consent}

The examination of the patient was conducted according to the Declaration of Helsinki principles.

\section{REFERENCES}

1. Lallas A, Kyrgidis A, Tzellos TG, Apalla Z, Karakyriou E, Karatolias A, et al. Accuracy of dermoscopic criteria for the diagnosis of psoriasis, dermatitis, lichen planus and pityriasis rosea. Br J Dermatol 2012;166:1198-205.

2. Zalaudek I, Argenziano G. Dermoscopy subpatterns of inflammatory skin disorders. Arch Dermatol. 2006;142:808.

3. Vazquez-Lopez F, Palacios-Garcia L, Gomez-Diez S, Argenziano G. Dermoscopy for discriminating between lichenoid sarcoidosis and lichen planus. Arch Dermatol. 2011;147:1130.

4. Gungor S, Topal IO, Erdogan S, Ozcan D. Classical lichen planus and lichen planus pigmentosus inversus overlap with dermoscopic features. Our Dermatol Online. 2014;5:42-4.

5. Vázquez-López F, Gómez-Díez S, Sánchez J, Pérez-Oliva N. Dermoscopy of active lichen planus. Arch Dermatol. 2007;143:1092.

6. Vázquez-López F, Vidal AM, Zalaudek I. Dermoscopic subpatterns of ashy dermatosis related to lichen planus. Arch Dermatol. 2010; 146:110.

Copyright by Aicha Nassiri, et al. This is an open-access article distributed under the terms of the Creative Commons Attribution License, which permits unrestricted use, distribution, and reproduction in any medium, provided the original author and source are credited.

Source of Support: Nil, Conflict of Interest: None declared. 